\title{
La couleur de Mai 1968
}

\section{Paris Match face aux événements de mai et juin 1968}

\author{
Audrey Leblanc ${ }^{1}$ \\ EHESS, Paris, France \\ Université Lille 3, France \\ https://clinoeil.hypotheses.org/
}

À l'appel de la grande manifestation unitaire du 13 mai 1968, le syndicat du livre auquel sont affiliés la plupart des ouvriers des imprimeries et des industries du papier rejoint le mouvement général de grève qui touche alors directement la presse magazine d'actualité. Les rédactions des magazines français L'Express, Le Nouvel Observateur et Paris Match sont ainsi confrontées à des problèmes d'impression et de distribution qui ont des répercussions immédiates sur leurs parutions. Les hebdomadaires réagissent différemment à ces mouvements sociaux et politiques qui affectent leur économie et le photojournalisme dit d'information. Si L'Express et Le Nouvel Observateur montrent une volonté d'adaptation et partagent une partie des fondements politiques de ces mouvements, Paris Match cesse de paraître pendant quatre semaines consécutives. Quand il reparaît le 15 juin, le magazine reprend le récit des événements là où il l'avait interrompu le 18 mai. Cependant, le statut de ces informations a changé : actualités à la mimai, elles sont à la mi-juin des événements du passé. Dans ce numéro de Paris Match du 15 juin, la rédaction propose une lecture rétrospective des événements du printemps 1968, racontés comme terminés. Ce récit est porté par un traitement formel en noir et blanc qui traduit - et défend - les intentions éditoriales du magazine soutenant le général Charles de Gaulle à la veille du premier tour des élections législatives de juin. Ce traitement formel en noir et blanc, inhabituel pour le magazine qui publie en principe en couleurs, met à jour les contraintes auxquelles la rédaction se confronte dans la conception d'un numéro. L'analyse des choix éditoriaux effectués dans le traitement des événements de Mai 1968 révèle ainsi les enjeux économiques et idéologiques de ce travail d'information.

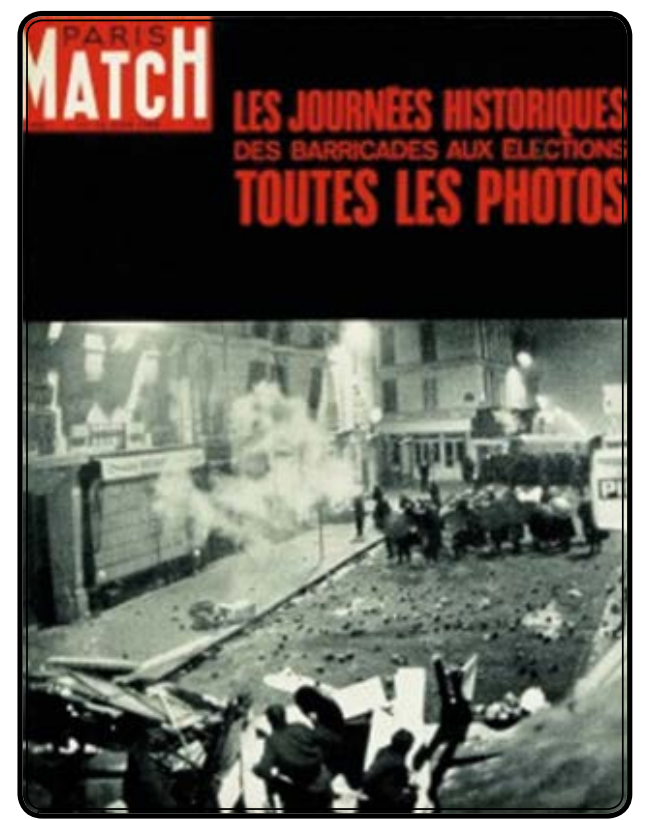

Figura 1. Paris Match n998, 15-22 juin 1968

1 Este artículo fue publicado inicialmente en 2010, Audrey Leblanc, "La couleur de Mai 1968 », Études photographiques, 26 I novembre 2010, [En ligne], mis en ligne le 08 juillet 2011. URL : http://journals.openedition. org/etudesphotographiques/3121.L'auteur tient à remercier, pour la richesse de leurs échanges en entretien Bernard Perrine, ancien rédacteur en chef du magazine Le Photographe; Dominique Brugière, laborantin à Paris Match entre 1965 et 1971 ; et Sébastien Dupuy, rédacteur en chef des collections et photographes Sygma Initiatives à Corbis; ainsi que Thierry Gervais, André Gunthert et Gaby David pour leurs relectures. La revista Entornos agradece a la profesora la revisión del texto y la autorización para incluirlo en este número de la revista. 


\section{Les habitudes éditoriales bouleversées}

Début mai 1968, l'ensemble de la presse française réagit vivement aux affrontements spectaculaires entre les étudiants et les forces de l'ordre qui ont lieu le 6 mai à la Sorbonne ${ }^{2}$. Ces événements apparaissent à la une des quotidiens et font l'objet de reportages de plusieurs pages dans la presse magazine d'actualité dominée alors par L'Express, Le Nouvel Observateur et particulièrement Paris Match. Chacun d'entre eux a ses habitudes éditoriales qui s'expriment notamment dans l'usage du noir et blanc ou de la couleur. Si L'Express et Le Nouvel Observateur présentent une dominante noir et blanc, Paris Match est très coloré. De grand format avec une couverture en couleurs, sa pagination varie entre 150 et 180 pages dont la moitié seulement est imprimée en noir et blanc. Un tiers du numéro se compose de publicités et le reste de reportages. Racheté par Jean Prouvost en 1938, le magazine sportif Match, devenu magazine d'actualité, reparaît en 1949 et marque d'emblée sa différence en choisissant la couleur. L'ancien journaliste Guillaume Hanoteau témoigne de ce choix, caractéristique d'après lui de l'audace propre à l'équipe du magazine : "Il avait été décidé, dans les Conseils de direction, que pour lancer Paris Match on consacrerait nombre de ses pages aux photos couleur. C'était une innovation. Le Match d'avant-guerre n'avait jamais utilisé cette couleur [...]. On voulut, néanmoins, en 1950, provoquer grâce à cette couleur une surprise et un choc. Ce n'était point sans témérité car la technique de ces photos était encore mal connue en France» ${ }^{3}$. Au moins un des reportages annoncés en couverture du magazine est illustré en couleurs dans les pages intérieures - traitement traduisant une place privilégiée dans la hiérarchie de l'information ${ }^{4}$. Pour le magazine, la maîtrise progressive des techniques couleur s'inscrit ainsi au cœur du traitement de l'actualité. Paris Match obtient un succès rapide avec un tirage trois fois supérieur à celui de ses concurrents et bénéficie d'une suprématie peu discutable auprès du lectorat en $1968^{5}$. Il est également important dans le milieu professionnel de l'époque, comme en témoigne Hubert Henrotte ${ }^{6}$, alors directeur de Gamma puis de Sygma à partir de 1973 - deux des plus importantes agences photographiques françaises. Dans ses mémoires, il mentionne rarement le titre des magazines dans lesquels sont publiés les reportages et les photographies vendus à la presse par l'agence, à l'exception notoire des publications dans Paris Match, systématiquement associées au succès, voire à la consécration, du photographe et même de l'agence ${ }^{7}$. Paris Match s'impose ainsi comme le magazine de référence au sein duquel il est

2 Philippe Artières et Michelle Zancarini-Fournel (dir.), Mai 68, une histoire collective [1962-1981], Paris, Éditions La Découverte, 2008, p. 215.

3 Guillaume Hanoteau, La Fabuleuse Aventure de Paris-Match, Paris, Plon, 1976. p. 39-40. Cf. Jean-Marie Charon, La Presse magazine, Paris, Éditions La Découverte, coll. "Repères", 2008 (1re édition, 1999), p. 13.

4 « Traiter un événement en couleurs, c'est lui donner une importance [...] la somme de toutes les contraintes est un frein naturel à son utilisation. Un traitement en couleurs relève du luxe ». Dominique Brugière laborantin à Paris Match entre 1965 et 1971 (en service militaire aux Antilles, janvier 1968-avril 1969). Entretien 1er octobre 2009, Paris.

5 « Très vite, les magazines d'actualité générale (Le Monde illustré, Point de vue, Images du monde) souffrent de la concurrence de Match [...] Le premier numéro reflète l'ambition de réaliser un hebdomadaire illustré moderne, à fort tirage, ouvert sur l'information mondiale et publié en couleurs. [...] [il] devient un hebdomadaire populaire de masse, sans équivalent dans les années 50 - le tirage atteignant 1,8 millions exemplaires en 1957», Thierry Gervais et Gaëlle Morel, “Les formes de l'information. De la presse illustrée aux médias modernes (1843-2002)", in André Gunthert et Michel Poivert (dir.), L'Art de la photographie, des origines à nos jours, Paris, Citadelles et Mazenod, 2007, p. 338. Dans les années 1960, les autres hebdomadaires d'actualités tirent à trois fois moins d'exemplaires (entre 300000 et 600 000). Jean-Marie Charon, La Presse magazine, op. cit., p. 13 (L'Express indique 512000 exemplaires pour le no 881 du 6 mai 1968, par exemple).

6 Jean-Louis Gazignaire et Hubert Henrotte (dir.), Le Monde dans les yeux. Sygma. L'âge d'or du photojournalisme, Paris, Hachette Littératures, 2005.

7 Ibid. p. 19, 21, 27, 60, 72 et chapitre 8 "Roger Thérond, le géant de Paris-Match" (p. 77-87). 
prestigieux et gratifiant de publier ses images; il domine la presse magazine d'actualité au printemps 1968 et se distingue par un usage conséquent de la couleur.

C'est après la première nuit des barricades dans le quartier latin les 10 et 11 mai que les trois magazines consacrent pour la première fois leur Une aux événements dits « étudiants», photographie de couverture à l'appui. Leur réaction synchrone et similaire (affrontements des étudiants et des forces de l'ordre comme l'indiquent les trois couvertures) signifie l'importance qu'ils accordent aux événements (fig. 2 à 4). Chacun d'entre eux aborde cette actualité dans le traitement éditorial - texte/ images/maquette - qui lui est habituel : reportage en noir et blanc dans Le Nouvel Observateur et L'Express; couverture en couleurs pour L'Express et Paris Match; un mélange d'iconographie couleur et noir et blanc pour Paris Match qui souligne sa différence en titrant "La révolte des étudiants - Couleur nos documents photo".

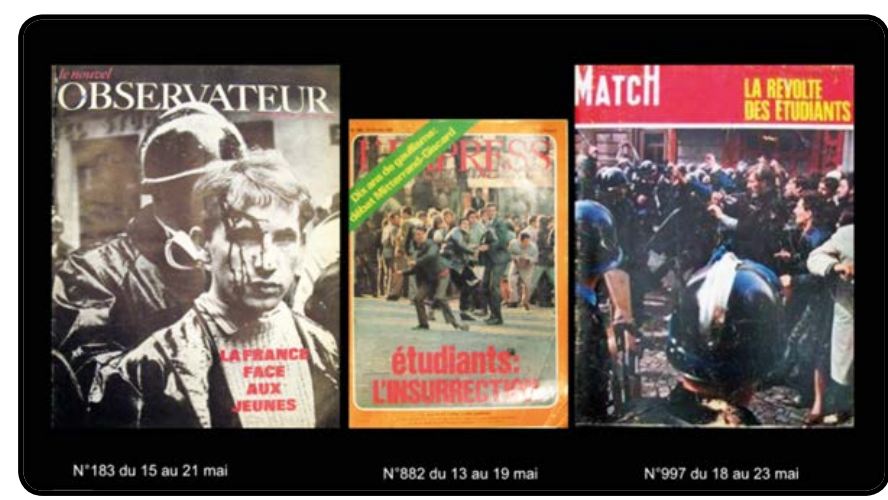

Figura 2 à 4

En réaction à ces affrontements pour le moins remarquables, les ouvriers rejoignent officiellement le mouvement de contestation pour la grande manifestation unitaire du 13 mai. À l'appel du syndicat du livre à large dominante CGT, les ouvriers du papier et des imprimeries se mobilisent eux aussi et entament un mouvement qui s'inscrit dans une longue tradition de luttes et de revendications. L'implication de ces travailleurs du livre et du papier dans le mouvement social général perturbe alors la chaîne de production de la presse, en particulier l'impression et la diffusion de la presse magazine ${ }^{8}$.

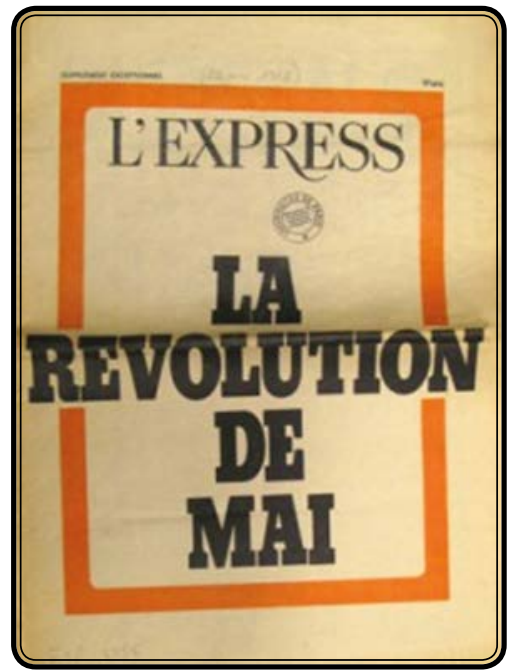

Figura 5. - L'Express $n^{\circ}$ spécial format quotidien

Les trois rédactions ne réagissent plus dès lors de la même façon face aux mouvements et leur appréhension des actualités s'en trouve modifiée. L'Express maintient sa parution hebdomadaire habituelle jusqu'au 20 mai (no 883), puis publie un supplément exceptionnel, au format des quotidiens et sur un papier similaire - quatre feuilles et une couverture en noir, blanc et rouge. Un encart de la rédaction explique ses perturbations de la publication liées aux mouvements de grève qu'elle soutient ${ }^{9}$.

8 Les grévistes maintiennent la presse quotidienne pour garantir la diversité des sources d'informations.

9 « Déclaration des collaborateurs de "L'Express". Le numéro de "L'Express" de cette semaine ne paraît pas, en raison des difficultés matérielles dans les imprimeries et les messageries. Dans les heures historiques que traverse la France, tous les collaborateurs du journal - employés, cadres et journalistes - ont tenu à être cependant présents, par la rédaction, l'impression et la distribution de ce supplément spécial, comme ils ont été présents à la manifestation populaire du 13 mai. Solidaires des aspirations fondamentales exprimées par les étudiants et les travailleurs en grève, tous les collaborateurs de "L'Express" ont estimé qu'il était de leur devoir de continuer à participer, par l'expression comme par l'action, au mouvement nouveau des idées et des hommes, en faisant paraître ce supplément exceptionnel». L'Express, p. 2 du supplément exceptionnel paru entre les nos 883, 20-26 mai et no 884, 17-23 juin 1968. 
Le magazine n'assure plus de parution régulière jusqu'au 17 juin. Le Nouvel Observateur maintient lui aussi sa parution hebdomadaire jusqu'au 22 mai. Retardé et intitulé "Le Grand Chambardement", le no 184 fait l'objet d'une explication de la part de la rédaction publiée dans un encart ${ }^{10}$. Le no 185 sort (quatre feuilles pliées soit 16 pages) daté du «jeudi 30 mai 1968», imprimé en Allemagne et présenté comme une édition spéciale. Le numéro suivant, daté du «vendredi 7 juin», paraît sans mention de lieu d'impression et avec un nombre de pages deux fois moins important que l'habituel (32 pages). Ce n'est que le 12 juin (no 187) que le magazine reprend son rythme régulier hebdomadaire.

De son côté, Paris Match cesse toute parution entre le 18 mai et le 15 juin. Lorsqu'il reparaît, la rédaction signe un encart exceptionnel aux côtés du sommaire qui explique l'augmentation $\mathrm{du}$ prix du numéro et donne l'occasion de rappeler les ambitions du magazine : il n'est pas fait référence aux raisons de sa longue absence dans les kiosques ${ }^{11}$. Si L'Express et Le Nouvel Observateur ont adopté une position de soutien des mouvements de grève, Paris Match ne les évoque que pour remercier ceux qui les ont soutenus dans ce qui a été manifestement vécu comme une épreuve ${ }^{12}$, l'allusion à un disfonctionnement dans les abonnements servant de seule mention de l'interruption de la publication.

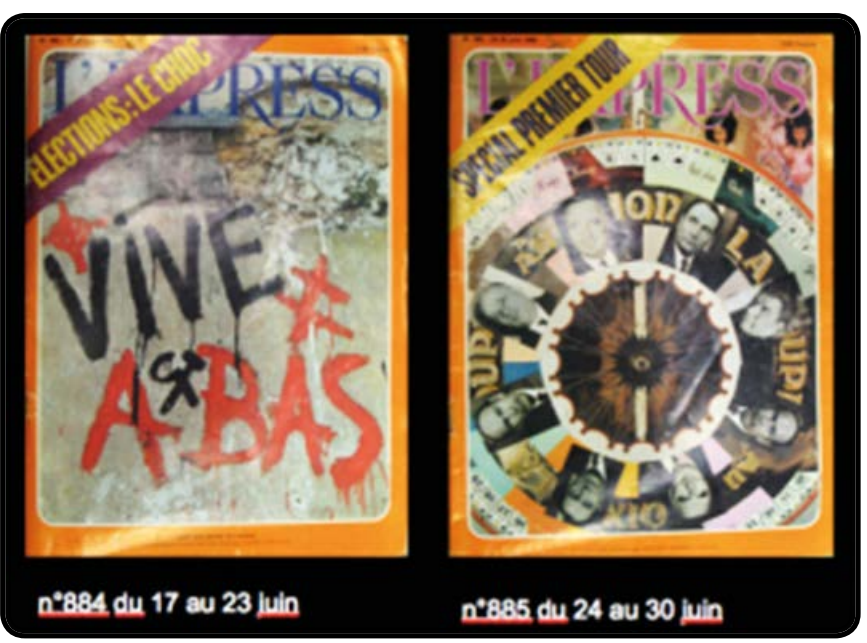

Figura 6 et 7. L'Express nº84, 17-23 juin 1968 n885, 24-30 juin 1968

Ainsi, les trois magazines reparaissent de nouveau lorsque les grèves dans les imprimeries cessent mi-juin. L'Express et Le Nouvel Observateur reprennent leur ligne éditoriale habituelle : ils prennent acte dès leur couverture des mouvements de grève alors en cours, de

10 «Les conséquences normales des événements actuels nous ont conduits pour la seconde fois à retarder la mise en vente du Nouvel Observateur. Notre solidarité avec l'immense mouvement national de contestation, et notre sympathie active pour les travailleurs intellectuels et manuels en grève sont telles que c'est avec sérénité que nous partageons le sort commun. Nous voulons pourtant remercier les centaines d'amis qui se sont émus à la pensée que nous puissions être mis dans l'incapacité de faire entendre notre voix à l'heure où les circonstances deviennent si graves, et après que nous ayons [sic] été l'un des rares journaux à informer objectivement l'opinion depuis le début des événements». Le Nouvel Observateur, no 184, 22-28 mai 1968, p. 23. 11 «Dès sa naissance, "Paris Match" a conçu une grande ambition. Tenant à l'écart la démagogie du sexe et du sang, celle de faire un magazine de haute culture apportant aux couches profondes de la France une image fidèle et noble du monde. "Paris Match" s'est également efforcé de rester accessible au plus grand nombre des Français en maintenant son prix de vente au niveau le plus bas possible compatible avec sa qualité. Les charges nouvelles contraignent "Paris Match" à élever son prix de vente à 2 francs. Nos lecteurs doivent être convaincus qu'il n'existe pas pour "Paris Match" une autre manière de rester fidèle à sa vocation et à sa mission. Nous avons été particulièrement sensibles aux témoignages de sympathie que nous avons reçus de nos lecteurs, nos annonceurs et de leurs conseils. Qu'ils en soient remerciés. [en plus petit] Nous nous excusons auprès de nos abonnés et naturellement leur service sera prolongé de quatre semaines». Paris Match, no 998, 22 juin 1968, p. 3. 12 "C'est peu dire que Mai 1968 fut une catastrophe pour Paris Match. À cause de la grève générale, l'hebdomadaire ne parut pas pendant quatre semaines, l'exceptionnelle moisson photographique restait au marbre et les reporters couvraient les événements, le moral à zéro, grattant pour rien : l'histoire se dessinait sous leurs yeux et ils se voyaient condamnés au silence ", Nicolas de Rabaudy, Nos Fabuleuses Années Paris Match, Paris, Scali Document, 2007, p. 168-169. Mai 68 marque aussi la fin de la collaboration de Jean Prouvost et Roger Thérond suite à un conflit à propos d'une éventuelle société de journalistes à Paris Match. 
l'approche des élections législatives à venir et d'une période marquée par le débat. En effet, L'Express titre "Vive \& à bas" pour son numéro du 17 juin, puis publie une photographie de tombola à la Une du numéro suivant. De son côté, Le Nouvel Observateur du 12 juin titre "Les insurgés de la Télévision", puis "Mendès-France, J-P Sartre, Kastler" (voir fig. 5 à 8). Dans son numéro du 15 juin, Paris Match se distingue dans le traitement de l'actualité et titre des "Journées historiques". Dès sa couverture, le magazine arbore de façon singulière une photographie en noir et blanc qui représente la première nuit des barricades du 10 mai : il reprend ainsi en guise $\mathrm{d}^{\prime}$ « actualités » des événements datant pour certains de plus d'un mois (voir fig. 1 et 9).

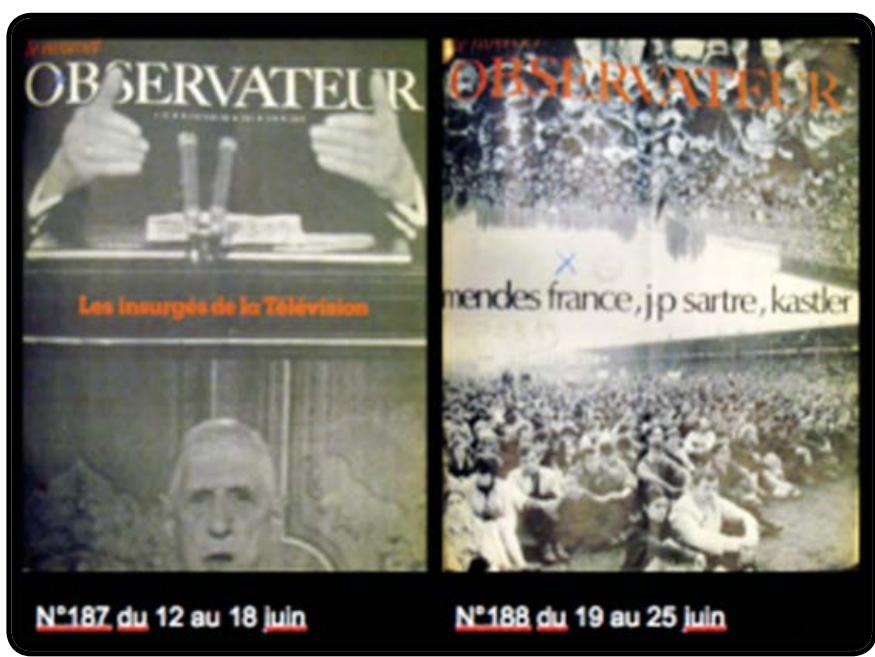

Figura 8 et 9. Le Nouvel Observateur, $\mathrm{n}^{\circ} 187,12-18$ juin 1968 et $\mathrm{n}^{\circ} 188,19-25$ juin 1968

\section{Reléguer les événements contemporains à l'Histoire}

La rédaction de Paris Match inscrit le $\mathrm{n}^{\circ}$ 998 du 15 juin dans la continuité éditoriale du précédent, datant du 18 mai. Un mois auparavant, elle proposait un reportage conséquent de 23 pages sur les événements "étudiants " (alors contemporains de la publication) en insistant sur les plus spectaculaires : la première nuit des barricades et la journée d'affrontements du 6 mai. Bien que des négociations soient en cours dans certaines usines, que certains mouvements de grèves se poursuivent (usines Renault ou Citroën, ORTF...) et que la campagne des élections législatives ait commencé, Paris Match propose mi-juin un numéro consacré aux événements du printemps 1968 construit sous la forme du bilan.

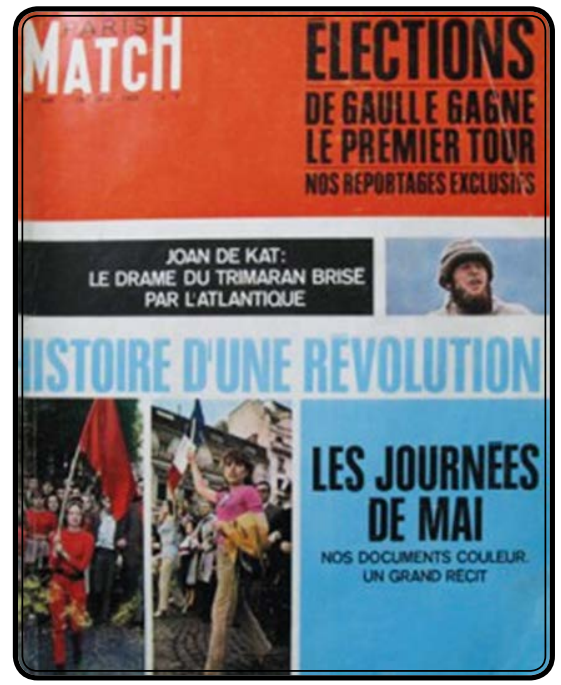

Figura 10. Paris Match n999, 23-29 juin 1968

En 1968, la maquette de Paris Match suit un ordonnancement précis qui peut être perturbé pour permettre à la rédaction de s'adapter aux besoins de l'actualité. Respectant la contrainte de l'impression par feuilles, des rubriques régulières se répondent de part et d'autre de la doublepage centrale du numéro, lieu de bascule. Le no 998 reprend cette structure qui est ajustée pour construire un numéro entièrement consacré aux événements de «Mai 68 ». Reléguant l'habituelle première série de publicités couleur et noir et blanc, la page de sommaire commence le magazine, immédiatement suivie par les pages de la rubrique "Le Match de la vie - Le Monde/la France" qui se caractérisent en principe par leur papier mat et leur rehaut rouge. Cette mise en forme a disparu et ces pages sont intégralement dédiées à ce que la rédaction titre : “Ces vingt journées incroyables de mai 1968 qui ébranlèrent la France et le gaullisme". Aucune autre actualité - y compris internationale - n'est, comme il est pourtant d'usage, abordée dans ces pages ${ }^{13}$.

13 Le déménagement de l'hôtel Lutetia à Choisy-le-Roi du Nord-Vietnamien Xuan Thuy, chef de la délégation de Hanoï, sert de référence à l'actualité internationale. 
De même, les six pages culturelles qui leur répondent à la fin du numéro - "Le Match de la vie - à Paris" - concernent les bouleversements dont les institutions culturelles ont fait les frais suite aux mouvements (festival de Cannes, Théâtre de l'Odéon, ORTF ${ }^{14}$...). Sur les 166 pages qui composent ce numéro, cinquante (pages 55 à 105) traitent ce que le sommaire annonce sous sa rubrique "Match actualité" : à savoir, le reportage sur "Les Journées historiques - des barricades aux élections". Enfin, plusieurs reportages se succèdent en principe au centre du numéro : dans celui-ci, seules huit pages sont consacrées au récit en images de l'assassinat de Robert Kennedy aux États-Unis le 5 juin $^{15}$. Organisé autour de soixante pages centrales (pages 55 à 114) de reportage, dont cinquante concernent «Mai 68», encadrées par autant de pages de publicités et par les rubriques habituelles qui ouvrent et referment le magazine, le numéro du 15 juin de Paris Match donne une place exceptionnelle ${ }^{16}$ aux événements du printemps, instituant leur importance et les monumentalisant.

Sous le titre "Les Journées historiques - des barricades aux élections", la rédaction propose le récit d'une période de transition révolue, qui inscrit les événements du printemps 1968 dans une dimension historique. Au 15 juin, « Mai 68 » est déjà passé à l'Histoire. En cinquante pages de reportage, la rédaction propose en neuf chapitres consécutifs et linéaires une rétrospective à la logique reconstruite de ces journées qualifiées d'historiques. L'orientation idéologique est claire et le récit remplit la promesse de la couverture, relatant le passage d'une situation définie comme anarchique et insurrectionnelle - les «barricades »-au retour à une démocratie ordonnée et digne de ce nom - les «élections » (fig. 10). Chacun de ces chapitres est ponctué de photographies en double-page et méthodiquement repris dans le sommaire. Après le rappel de la première nuit des barricades du 10 au 11 mai, le récit s'organise ainsi : «Grand défilé du 13 mai (1), la grève s'étend (2), La Sorbonne ouverte à tous (3), Les jeunes redescendent dans la rue (4), Accords rue de Grenelle (5), Le gouvernement est sombre (6), Marée tricolore aux Champs-Élysées (7), Flins, bataille dans les blés (8), Encore une "nuit terrible $^{17 "}$ (9) ». Chaque épisode est construit autour d'événements choisis et distingués par la rédaction. Le nombre de pages et de photographies qui leur est consacré ainsi que la mise en page des photographies sont autant d'indicateurs de l'importance que leur accorde la rédaction. Avec dix doubles-pages - presque la moitié du reportage -, l'ensemble insiste sur les nuits d'affrontements spectaculaires et sur la dimension contestataire, voire subversive, des revendications étudiantes. Deux doublespages retracent l'intégralité des mouvements de grève sous le titre générique "La grève s'étend" et insistent sur l'échec de la jonction entre les étudiants et le monde du travail et sur celui des accords de Grenelle. D'autre part, le récit rétrospectif valorise la figure de Charles de Gaulle. La photographie-scoop, par Henri Bureau, du président à l'héliport d'Issy-lesMoulineaux le 29 mai 1968 lors de son retour de Baden-Baden après sa " disparition ", est publiée seule, en double-page, à la suite de celle représentant le gouvernement en plein désarroi à l'Assemblée nationale. Cetagencement des pages écrit le retour du président comme la réponse aux errances politiques du gouvernement. Les trois doubles-pages suivantes sont consacrées à la manifestation de soutien au président organisée le 30 mai et confirment l'orientation politique du récit. Les événements postérieurs au 30 mai sont ensuite mentionnés sous la forme de sursauts dérisoires. L'ensemble se clôt sur une photographie occupant les deux tiers de la double-page qui montre des jeunes dansant autour d'un feu nocturne sous la légende "La campagne électorale débute par une danse autour de panneaux en feu". Faisant

14 À une demi-page près, consacrée au tournoi de Roland-Garros.

15 Trois autres pages, proches d'un publireportage et mêlées aux publicités, vantent la nouvelle usine Pernod à Marseille, p. 13-15.

16 "Paris-Match sort enfin une édition spéciale, sur ce mois fou-fou-fou", Jean Durieux et Patrick Mahé, Les Dossiers secrets de Paris Match, Paris, Robert Laffont, 2009, p. 135.

17 Paris Match, no 998, 15 juin 1968, p. 55. 
écho aux photographies des barricades en feu, l'image crée la confusion entre les deux gestes et suggère l'irrespect des jeunes à l'égard de la démocratie. C'est une photographie en doublepage montrant Daniel Cohn-Bendit devant la porte de Brandebourg à Berlin, valise à la main, et légendée "Et maintenant il part prêcher l'anarchie à travers l'Europe", qui ferme cette rétrospective, à la veille du premier tour des élections législatives du mois de juin 1968. Sans mention des débats politiques en cours liés aux mouvements, le principe de la rétrospective et sa construction rendent compte d'un épisode décrit et montré comme subversif mais terminé ${ }^{18}$.

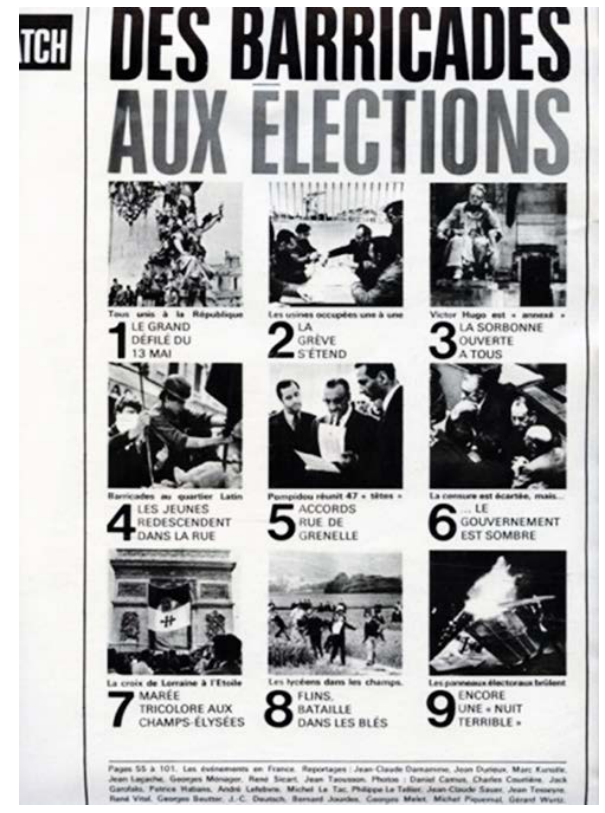

Figura 11. Sommaire de Paris Match n998, 15-22 juin 1968

Ces partis pris idéologiques lisibles dans la construction du récit sont soutenus par leur mise en forme ${ }^{19}$. Plusieurs fois convoquée dans les articles (en particulier dans le numéro précédent du 18 mai), la guerre d'Algérie fait office de point de comparaison à la " révolte étudiante» ${ }^{20}$. Un parallèle qui n'est pas anodin dans un moment où les légitimités présidentielle et gouvernementale sont remises en cause. Le no 998 du 15 juin mobilise aussi cette référence politique dans le choix de sa maquette. La couverture reprend en effet la construction graphique du numéro consacré à "La rébellion d'Alger", à savoir un large bandeau noir sur lequel s'affiche le titre en capitales accompagné d'une photographie noir et blanc ${ }^{21}$ (voir fig. 1 et 12 ).

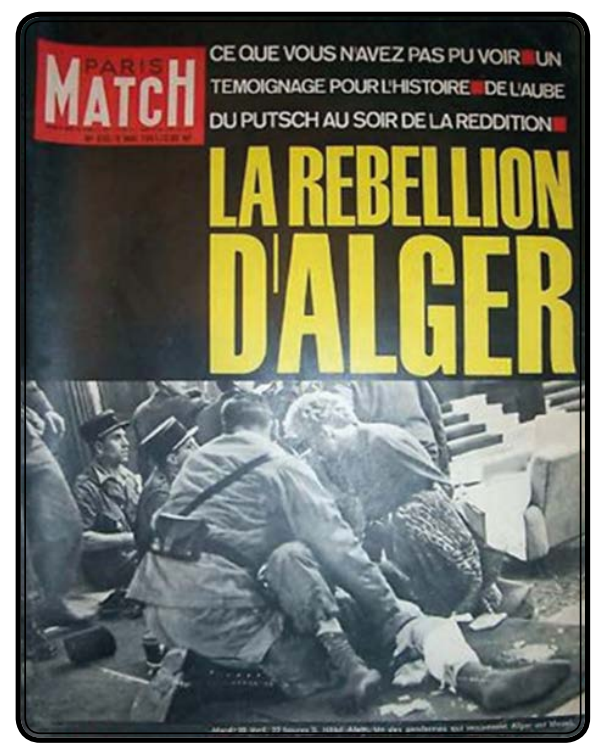

Figura 12. Paris Match n630, 6-12 mai 1961

La similitude formelle génère un rapprochement entre ces événements que les titres soutiennent également. Le numéro du 6 mai 1961 avait titré “Ce que vous n'avez pas pu voir. Un témoignage pour l'histoire. De l'aube du putsch au soir de la reddition. La Rébellion d'Alger". La tentative manquée de coup d'État par une partie des militaires de carrière de l'armée française en Algérie fin avril 1961 est désormais surnommée «le putsch des Généraux» ou le «Putsch d'Alger». Dans ce numéro du 6

18 La construction rétrospective permet aussi d'utiliser le matériel photojournalistique qui s'est accumulé au cours des événements, répondant ainsi à la nécessité économique du magazine et à la frustration professionnelle. Cf. note 11 .

19 Selon une expression de Thierry Gervais, in L'Illustration photographique. Naissance du de l'information (1843-1914), thèse en Histoire et Civilisation, EHESS, 2007, chapitre 4, "L'invention du magazine, les nouvelles formes de l'information (1898-1914)". En ligne : http://culturevisuelle.org/blog/4356, consultée le 19 mai 2010. 20 Titre du no 997 du 18 mai 1968.

21 Cette maquette de couverture est utilisée à deux autres reprises. Cf. note 21. 
mai, la rédaction de Paris Match annonce qu'elle propose des images et une lecture historique $\mathrm{du}$ putsch sous la forme d'une rétrospective. Le terme " rébellion », discutable d'un point de vue historique mais choisi pour cette couverture en 1961, favorise le rapprochement idéologique - et non plus seulement formel - mis en place entre la tentative de putsch et les événements de « Mai 68 », présentés sous l'angle de la révolte et de l'insurrection. Textes, maquette et images ne font qu'un dans l'élaboration d'un récit partisan des événements. En pages intérieures du no 998, l'unique reportage qui accompagne les cinquante pages consacrées aux événements du printemps 1968 et qui rend compte de l'assassinat de Robert Kennedy propose, dans ce contexte, la version tragique de ces « rébellions » auxquelles le pouvoir politique est confronté. Ces rapprochements font ainsi de la guerre d'Algérie et de "Mai 68 » deux épreuves rendues équivalentes par la similitude de leur traitement - auxquelles le pouvoir gaulliste a dû faire face. Le noir et blanc de la photographie de couverture confirme cette assise formelle du récit proposé des événements : pour le magazine, elle relève d'un usage rarissime qui traduit toujours son insistance sur la dimension historique des événements traités ${ }^{22}$. L'ensemble du no 998 concourt ainsi à l'historicisation mortifère d'événements pourtant encore vivaces et témoigne $\mathrm{du}$ soutien de la rédaction au général de Gaulle, à la veille du premier tour des élections législatives.

\section{Convertir la contrainte technique en atout éditorial}

Dans le contexte de grève des imprimeries, la question du traitement formel en couleurs ou en noir et blanc se révise à l'aune de contraintes techniques imposées à la rédaction par les conséquences des mouvements sociaux.

Dans le no 998 du 15 juin, seules des publicités ayant déjà été publiées auparavant par le magazine (qui dispose ainsi de leur maquette $d^{\prime}$ impression) sont en couleurs. Le rehaut rouge des rubriques d'actualité "Le Match de la vie" a disparu et l'iconographie des deux reportages d'actualité proposés est exclusivement noir et blanc. Ce traitement est inhabituel dans le magazine qui publie des photographies couleur pour souligner les événements considérés importants et qui dispose par ailleurs de nombreuses photographies couleur de ces événements ${ }^{23}$. L'imprimerie Chaix-DesfossésNéogravure, avec laquelle le groupe presse Prouvost travaille en 1968 - à savoir, la maison mère implantée à Issy-les-Moulineaux-, est l'une des plus importantes imprimeries de l'époque :

" La Néogravure, dans les années 19701980, est une imprimerie polygraphique: on y utilise aussi bien le procédé d'héliogravure que celui d'offset. Imprimerie de labeur, elle n'imprime pas de presse quotidienne [...] elle produit surtout des "périodiques". Citons pour mémoire Elle, Marie-Claire, Paris-Match, Lui, Play Boy, Le Chasseur français, Le Catalogue de la Redoute... Elle est à l'époque la première imprimerie française, la troisième européenne et possède le matériel d'héliogravure le plus moderne d'Europe» ${ }^{24}$.

L'imprimerie entre en grève à la mi-mai comme le mentionne un communiqué du 13 mai 1968 (CGT - FSM), signé des «Élus des deux collèges des Établissements de St-Ouen et d'Issy». Le

22 Voir les Unes de Paris Match depuis 1949 : http://paiement.parismatch.com/commande_numero/journal_ commander.php?texte $=1968 \&$ separ $=$ OR\&encadrement $==\&$ champs $0=05 / 08 / 2008 \& \mathrm{x}=0 \& y=0$, consulté le 1 er mai 2010.

23 Paris Match en a publié dans le no 997 du 18 mai et en publie de nombreuses dans les nos 999 et 1000 suivants, qui donnent aussi une place prépondérante aux événements (fig. 9).

24 Inventaire de Robert Codineau (en 1981-1982) d'une partie des archives du Comité d'entreprise de la Néogravure (1946-1979), consultables au Centre d'histoire sociale du xxe siècle, université Paris 1 ; bulletin du Centre, no 6, 1981-1982, p. 87-103 ou en ligne : http://histoire-sociale.univ-paris1.fr/Document/gravure.htm , consultées le 31 janvier 2010 
conflit s'accentue suite aux «accords de Grenelle» à la fin mai $^{25}$ et les premiers protocoles d'accord datent d'abord des 8 et 9 juin, puis des 14 et 15 juin, date exacte de la reparution de Paris Match avec le no 998 (fig. 12).

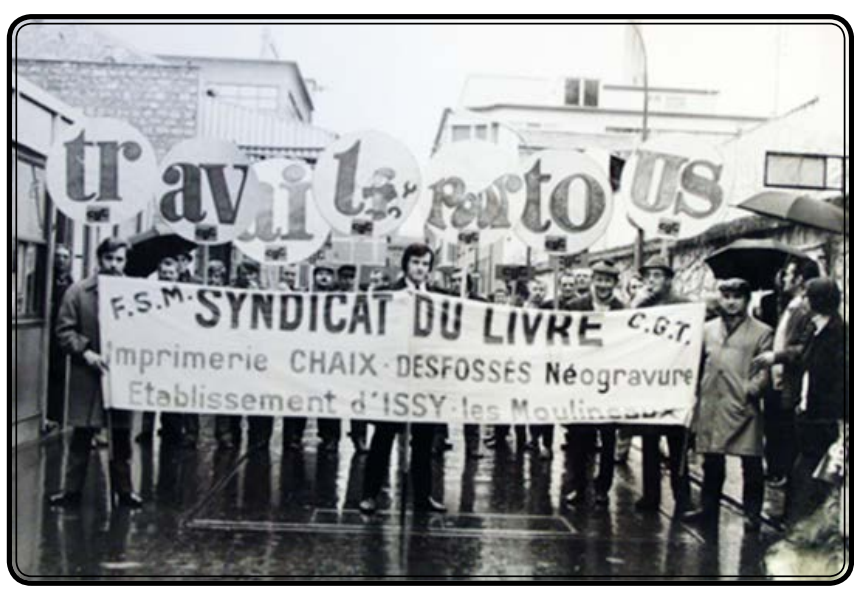

Figura 13. Anon. "Ouvriers de l'imprimerie Chaix-Desfossés-Néogravure en grève ", tirage argentique, $12 \times 18 \mathrm{~cm}$, archives du comité central de la néogravure. Coll. Centre d'Histoire sociale du XXè siècle, université Paris 1

La liste des postes de l'imprimerie indique que le traitement couleur, pratiqué au sein de la même entreprise, ne correspond pas aux mêmes cellules de travail que le traitement noir et blanc. L'impression noir et blanc reprend la première alors que l'impression couleur, requérant plus de soin, est stoppée : c'est ce problème technique qui contribue à expliquer l'exception de l'iconographie du no 998.

Lorsqu'il reparaît mi-juin, Paris Match doit composer avec un traitement noir et blanc de ses actualités qui lui est techniquement imposé et qui contrarie ses habitudes et sa force éditoriales. Cette contrainte technique aux conséquences formelles s'ajoute aux enjeux économiques (quatre semaines d'interruption de parution) et idéologiques (la volonté de soutenir le général de Gaulle) de cette reparution. L'élaboration du no 998 par le choix d'une rétrospective sur cinquante pages correspond à une réaction éditoriale pragmatique à un ensemble d'impératifs. Elle permet d'utiliser le matériel photojournalistique qui s'est accumulé pendant les semaines $\mathrm{d}$ 'interruption. Elle autorise également une mise en récit historique d'événements contemporains qui propose, comme un aboutissement logique, la confirmation du général de Gaulle dans ses fonctions. Elle permet enfin de donner un sens à la contrainte formelle du traitement noir et blanc. Inhabituel pour Paris Match, l'impression en noir et blanc de ce numéro spécial « Mai 68 » devient, dans cette construction de l'actualité, la couleur de l'Histoire $^{26}$. Dès lors, le noir et blanc n'est plus perçu comme une contrainte technique, mais comme une forme opportune pour la rédaction dans sa mise en récit des événements. La contrainte du noir et blanc, a priori fragilisante pour le magazine, s'est transformée en atout éditorial et ce disfonctionnement ponctuel est devenu l'indice du travail de mise en forme de l'information par la rédaction du magazine.

"Toutes les photos" titre le no 998 du 15 juin 1968 de Paris Match. La rédaction du magazine ne parle pas de l'impératif technique, accidentel et contrariant, à l'origine du traitement formel qu'elle propose des événements : elle l'assume en le faisant fonctionner avec l'ensemble des autres décisions présidant à la conception $\mathrm{du}$ numéro. Choix par défaut, il rend alors perceptible la complexité d'un ensemble : le magazine. L'iconographie accidentellement noir et blanc devient l'un des éléments qui ratifient et portent, au sein d'une construction complexe textes, images et maquette -, une interprétation idéologique des événements. En les racontant sous la forme d'un récit rétrospectif bien ordonné, déjà commémoratif et porté par une iconographie

25 Fin mai-début juin, le nombre d'appels et de documents relatifs à la mobilisation se multiplie. Le conflit est toujours désigné par l'expression « mouvement de mai-juin 1968 ».

26 Une même construction n'aurait pas fonctionné dans L'Express ou Le Nouvel Observateur, habitués à l'usage exclusif d'une iconographie noir et blanc. De plus, la photographie en une exceptionnellement noir et blanc inscrit ce numéro, dès sa couverture, dans la lignée de quelques numéros précédents (voir note 21), en Une forme éditoriale ponctuellement utilisée par le magazine. 
et une forme lisibles dans un tel contexte comme historicisantes, c'est la forme magazine ${ }^{27}$ tout entière de ce numéro de Paris Match qui renvoie Mai 68 dans les filets de l'histoire. "Toutes les photos", à connaître et à garder en mémoire sur « Mai 68 ", pourrait-on ajouter. Proche d'un album souvenir des événements, quel impact, quelle persuasion et quelle pérennité une forme médiatique à l'efficacité formelle si cohérente, portée ici par le magazine d'actualité français le plus important et le plus influent de l'époque, peut-elle effectivement avoir sur l'interprétation de ces événements historiques?

27 C'est-à-dire le dispositif médiatique global formé par le récit proposé des événements et les choix formels avec lesquels il s'imbrique. Voir l'éditorial d'André GUNTHERT et Thierry Gervais, "Les Images publiques ont une histoire", Études Photographiques, "La Trame des images. Histoire de l'illustration photographique", no 20, juin 2007, p. 2-3 (en ligne : http://etudesphotographiques.revues.org/index894.html , consulté le 19/05/2010), et T. Gervais, L'Illustration photographique..., op. cit., p. 404 et p. 418-452 (en ligne : http://culturevisuelle.org/ blog/4356, consulté le 19/05/2010). 


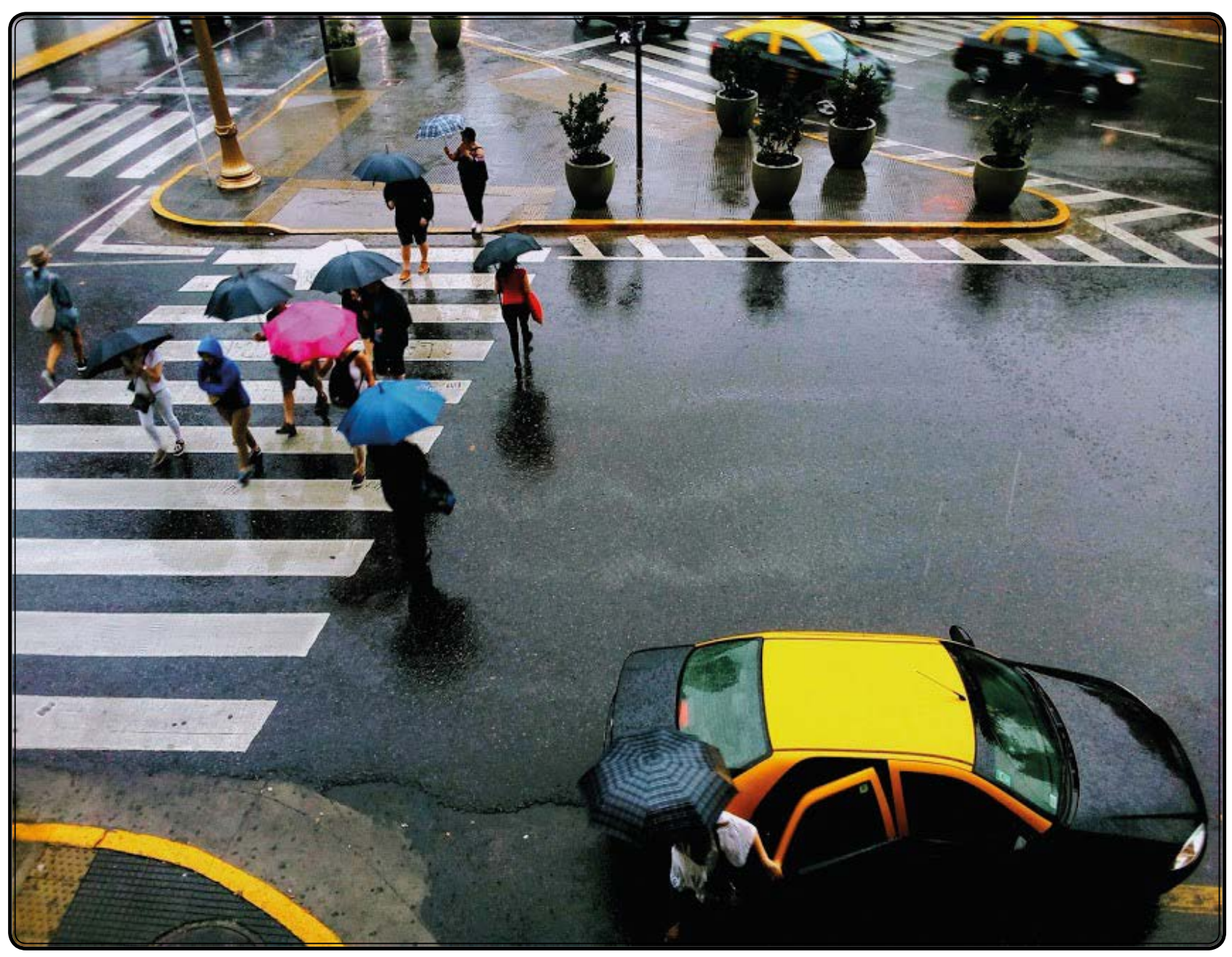

Lunares del desconsuelo. 